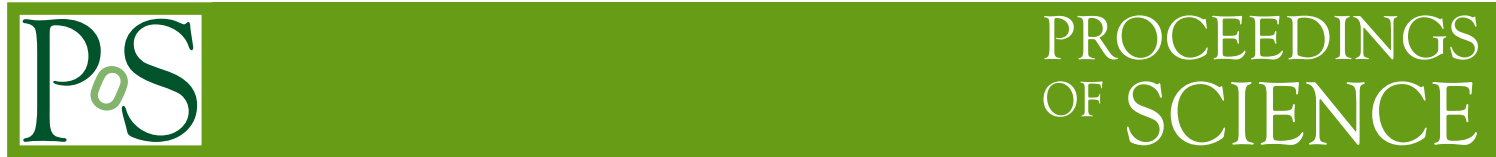

\title{
Non-linear QCD Dynamics and Wilson Lines
}

\author{
Giovanni Antonio Chirilli*† \\ Department of Physics, The Ohio State University, Columbus, OH 43210, USA \\ E-mail: chirilli.1@osu.edu
}

\begin{abstract}
The growth of the hadronic and nuclear scattering cross-sections while increasing the center-ofmass energy is described by the linear BFKL evolution equation. At very high energies, when the parton density becomes high, the cross-section approaches the unitarity limit, and the dynamics is described by the non-linear BK and JIMWLK evolution equations. These equations are currently the starting point of discussion for future experiments like the proposed Electron Ion Collider (EIC) or the Large Hadron electron Collider ( $\mathrm{LHeC}$ ), and play also an important role in current RHIC and LHC experiments involving nucleus-nucleus and proton-nucleus collisions.
\end{abstract}

QCD Evolution 2015 -QCDEV2015-

26-30 mAY 2015

Jefferson Lab (JLAB), Newport News Virginia, USA

\footnotetext{
* Speaker.

${ }^{\dagger}$ The author thaks the organizers of the QCD evolution 2015 workshop for warm hospitality.
} 


\section{Introduction}

Understanding the fine structure of matter is still a challenge in Quantum Chromodynamics. It is well know that the dynamics of hadronic matter at high-scattering energy is non-linear. The evolution equation that describes the growth of partons density is the BFKL equation. It predicts that the cross-section, in Deep Inelastic Scattering (DIS), grows as a positive power of the centerof-mass energy. This prediction, at very high energy, violates the unitarity constraint.

It is then an open question whether there exists a saturation limit for parton density in hadronic matter when this is probed at very high energy. It is known that the cross-section for scattering process off a disc of radius $R$ is bounded by: $\sigma_{t o t} \leq 2 \pi R^{2}$. Moreover, to properly describe highenergy scattering processes one has to know the proper degree of freedom i.e. what are the relevant operators of the theory.

High-energy Operator Product Expansion (OPE) of the scattering amplitude in terms of infinite Wilson line operators has been shown to be very successful [1,2]. Evolution equations that restore unitarity are the BK $[4,5]$ equation and the JIMWLK [6] equation. The BK equation is an evolution equation for dipole (Wilson lines) operators and is relevant for the description of DIS at highenergy. On the other hand, the JIMWLK equation, equivalent to the Balitsky's hierarchy of coupled evolution equations, is relevant for the description of processes like proton-Nucleus or NucleusNucleus collisions. The Balitsky-hierarchy is also relevant for DIS at higher order corrections i.e when DIS is described not only by dipole operators but also by product of multiple dipoles operators.

In Section 2 we give a brief introduction to the Leading Log Approximation technique and introduce the saturation scale while in Section 3 we describe parton dynamics and the saturation limit. A brief pedagogical introduction to the background field technique is given is Section 4, where we will derive, as an example, the evolution of one Wilson line operator. In section 6 we will present the NLO correction to the evolution equation of one Wilson line and the evolution equation of Wilson lines with triple interactions. The complete result of the Balitsky-JIMWLK evolution equation at NLO can be found in Ref. [9].

\section{Leading-Log-Approximation and the Saturation scale}

When the center-of-mass-energy $s$ is very high and the strong coupling constant $\alpha_{s}$ is small, contributions proportional to $\alpha_{s} \ln s$ represent the leading contribution in the theory and need to be resummed through an evolution equation, the BFKL equation. A sample of diagrams that are resumed in the Leading Log Approximation (LLA) by the BFKL equation are shown in Fig. (1).
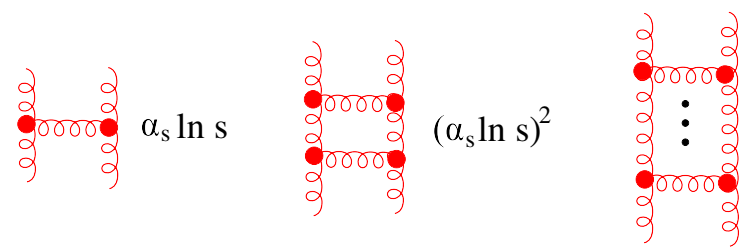

n $\left(\alpha_{\mathrm{s}} \ln \mathrm{s}\right)^{\mathrm{n}}$

Figure 1: Rung-type diagrams resummed in the Leading Log Approximation (LLA) by the BFKL equation. 
When the center-of-mass energy $s$ is much larger than all other scales involved in the scattering process, the use of perturbation theory is possible because there exists an intrinsic scale (the saturation scale), which, although much smaller than the center-of-mass energy, is well above the typical confinement region. An heuristic derivation of the saturation scale at high-energy is provided by the dipole-dipole (onium-onium) cross-section. In Fig. (2) is shown a typical diagram that contributes to the cross-section.

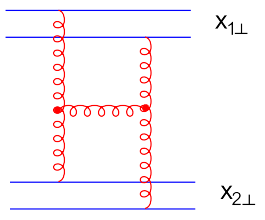

Figure 2: Onium-onium scattering process. This is a typical rung diagram in the LLA. The red bullet at the three-gluon vertex represent a Lipatov-effective vertex.

The cross section, obtained in the LLA, is proportional to the size of each dipole, $x_{1 \perp}, x_{2}$, to $\alpha_{s}^{2}$ due to the two-gluon exchange between the two dipoles, and to the exponential of energy (or rapidity $Y$ ) provided by the solution of the BFKL equation

$$
\sigma_{\text {tot }}^{\text {onium-onium }} \propto \alpha_{s}^{2} x_{1 \perp} x_{2 \perp} e^{\Delta Y}
$$

where $\Delta=\frac{4 \alpha_{s} N_{c}}{\pi} \ln 2>0$. The upper bound of the cross-section is the black-disk limit: $\sigma_{\text {tot }} \leq 2 \pi R^{2}$.

The cross section of onium-onium scattering (2.1) can be appropriately modified for the DIS case. Assume that one the two dipoles in Fig. 2 is the quark-antiquark pair produced by the virtual photon long before scattering off a proton or a large nucleus, and that the second dipole is the hadronic target with typical size $\Lambda_{Q C D}^{-1}$. The size of the quark-antiquark pair, instead, is $Q^{-1}$ where $Q=\sqrt{-q^{2}}$ and $q^{\mu}$ the momentum of the virtual photon. When DIS cross-section reaches the black-disk limit we obtain the typical momentum scale $Q_{s}$ at which the parton-density saturates

$$
Q_{s} \sim \alpha_{s}^{2} \Lambda_{Q C D}\left(\frac{1}{x_{B}}\right)^{\Delta}
$$

where $x_{B}$ is the Bjorken variable. As we can see from Eq. (2.2), $Q_{s}$ grows with energy. When $Q_{s}$ is larger then non-perturbative scale we may apply perturbative techniques.

\section{Parton Saturation and Color Glass Condensate}

In DIS processes, as is shown in Fig. 3, when the target is boosted at higher and higher energy, the number of partons available for the interaction with the projectile i.e the dipole, increases, and consequently the cross section does too.

If the power resolution of the projectile (see Fig. 4), which is the inverse of the momentum transverse to the target, is kept fixed while we increase the energy of the system, the density of the partons increases. The reason is that partons that are resolved with fixed transverse size increases in number. Consequently, the system evolves towards a saturation limit (Color Glass Condensate) where the density cannot grow further because unitarity cannot be violated. The mechanism that 

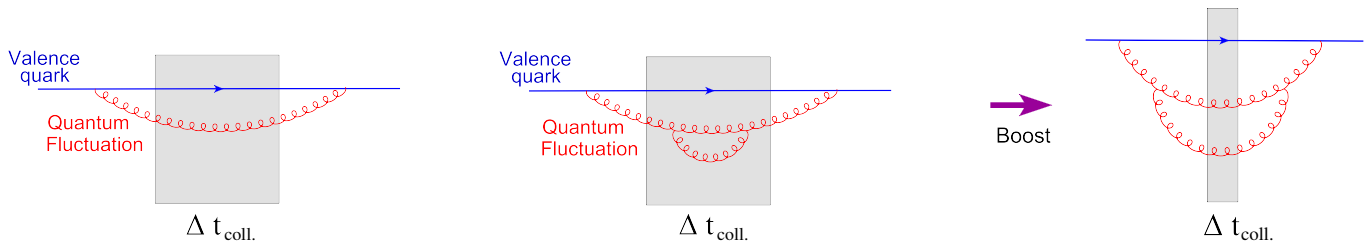

Figure 3: In this figure the lifetime of the projectile, depicted as a gray bend, is compared with the lifetime of the quantum fluctuations. In the left panel it is shown the case in which the lifetime of the projectile is comparable to the lifetime of the quantum-fluctuation. In this case the interaction projectile-quantum fluctuation may happen. In the center panel, it is shown a second fluctuation laying entirely in the gray bend thus having a lifetime much shorter then the lifetime of the dipole. In this case the dipole will not be able to interact with the fast quantum fluctuation. In the right panel it is shown the enhancement of the lifetime of the quantum fluctuations after performing a boost of the target. In this case the dipole may interact with both quantum fluctuations.

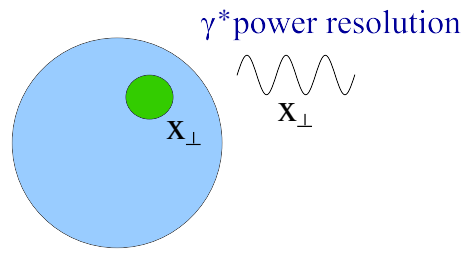

Figure 4: The power resolution of the projectile is given by the wave-length of the virtual photon and consequently by the size of the quark-antiquark pair. In this figure a parton (in green) inside an hadronic target (in blue) is resolved by the projectile since they are of the same transverse size.

tames the growth of parton-density is recombination. When the density is very high, the phasespace is saturated (see Fig. 5), and partons start a recombination process which makes the dynamics of the system to be non-linear. The description of non-linear dynamics of parton's evolution is provided by non-liner equations. As we will see in the next section, evolution equation for the description of parton-density in the saturation limit are in terms of Wilson lines operators.
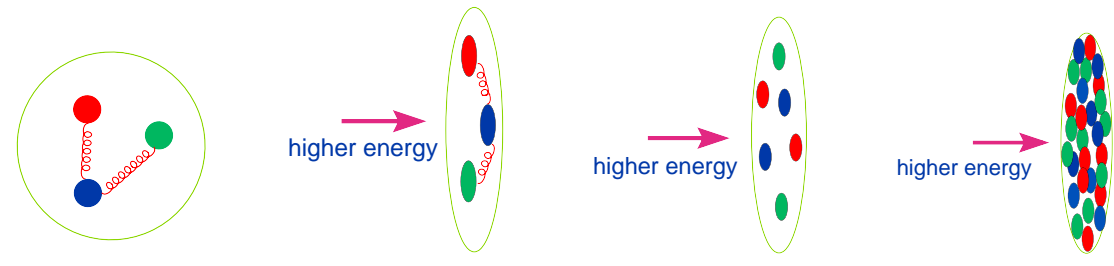

Figure 5: In this picture is shown that the density of parton in the hadron increases after each consecutive boost until it reaches a limit where the density cannot grow any further. This is the saturation limit.

\section{Evolution equation of Wilson lines in QCD}

Wilson line operators are path-ordered exponential of the gauge field along the trajectory of 
the particle's velocity. In QCD the Wilson lines are defined as

$U\left(x_{\perp}\right)=1+i g \int_{-\infty}^{\infty} d u A_{\bullet}\left(u p_{1}+x_{\perp}\right)+(i g)^{2} \int_{-\infty}^{+\infty} d u \int_{-\infty}^{u} d v A_{\bullet}\left(u p_{1}+x_{\perp}\right) A_{\bullet}\left(v p_{1}+x_{\perp}\right)+\ldots$

Here, we use the notation $x_{\bullet}=\sqrt{\frac{s}{2}} x^{-}$and $x_{*}=\sqrt{\frac{s}{2}} x^{+}$with $x^{+}$and $x^{-}$light-cone coordinates defined as $x^{ \pm}=\frac{x^{0} \pm x^{3}}{\sqrt{2}} ; p_{1}^{\mu}$ and $p_{2}^{\mu}$ are light-cone vectors such that $p_{1}^{2}=p_{2}^{2}=0$ and $p_{1} \cdot p_{2}=\frac{s}{2}$ with $s$ the Mandelstam variable for the center-of-mass energy.

At High-energy, scattering amplitudes can be expanded in terms of Wilson lines using the Operator Product Expansion (OPE) [4, 7]. The energy dependence of the amplitudes is provided by the evolution equations in rapidity of the Wilson line operators.

As explained in the previous section, In DIS the virtual photon emitted by the lepton long before scattering off the hadronic target, splits into a quark and anti-quark pair. The propagation of the color dipole through the hadronic target is given by a scattering amplitude proportional to two Wilson lines. Thus, we can write the amplitude as a convolution of the a coefficient function (the photon impact factor now known at NLO [1,2]) and a matrix element of a color dipole. The first of the Balitsky's hierarchy of evolution equations is represented by the evolution equation of the color dipole. In the large $N_{c}$ approximation this evolution equation reduces to the Balitsky-Kovchegov equation $[4,5]$ (for a review see Ref. [8]).

Relevant operator for the description of scattering amplitude for proton-Nucleus or NucleusNucleus collisions are operators made of traces of several Wilson lines. The evolution equation of such operators correspond to the Balitsky-JIMWLK evolution equation $[4,6]$.

In this section we provide an introduction to the background field method used to derive the evolution equation of Wilson lines.

At high-energy (high-parton density) the energy dependence of the scattering amplitude is encoded in the evolution of matrix elements made of Wilson lines. Let us indicate with $\mathscr{O}^{\eta_{1}}$ an operator made of several Wilson lines with rapidity dependence $\eta_{1}$. If we indicate with $|B\rangle$ the target state, then the scattering amplitude is proportional to

$$
\left\langle B\left|\mathscr{O}^{\eta_{1}}\right| B\right\rangle
$$

The Wilson lines may depend on the rapidity parameter in at least two different ways. One way is the dependence by slope: if the particle propagate at infinite energy then its trajectory is on the light cone. If we assume that the energy is very large but not infinite, then, the trajectory of the particle is slightly off the light cone i.e it is along $n^{\mu}=p_{1}^{\mu}+e^{-2 \eta} p_{2}^{\mu}$ direction. The energy of the particle is given by its rapidity $\eta$ and when the energy is infinite the propagation of the particle is parallel to the light-cone vector $p_{1}^{\mu}$.

The rapidity dependence by slope of the Wilson line is

$$
U^{\eta}\left(x_{\perp}\right)=\operatorname{Pexp}\left\{i g \int_{-\infty}^{\infty} d u n_{\mu} A^{\mu}\left(u n+x_{\perp}\right)\right\}
$$

Alternatively, one can include the energy dependence into the Wilson line operator by rigid cut-off i.e. by cutting off the longitudinal component of the momentum of the gluon in the following way

$$
U_{x}^{\eta}=\operatorname{Pexp}\left[i g \int_{-\infty}^{\infty} d u p_{1}^{\mu} A_{\mu}^{\eta}\left(u p_{1}+x_{\perp}\right)\right]
$$


with

$$
A_{\mu}^{\eta}(x)=\int \frac{d^{4} k}{(2 \pi)^{4}} \theta\left(e^{\eta}-\left|\alpha_{k}\right|\right) e^{-i k \cdot x} A_{\mu}(k)
$$

and with $k^{\mu}=\alpha_{k} p_{1}^{\mu}+\beta_{k} p_{2}^{\mu}+k_{\perp}^{\mu}$. As it has been shown in Ref. [12, 9], at NLO it is more convenient to use the rapidity dependence by rigid cut-off.

At high energy the main degree of freedom are gluons, therefore we may assume, in the first approximation, that the hadronic target is made of gluon field. We consider, then, the operator $\mathscr{O}^{\eta_{1}}$ in the background of an external gluonic field and we want to calculate its evolution with respect to the rapidity parameter by changing the parameter $\eta_{1}$ by an infinitesimal step $\Delta \eta$. To this end, we need to introduce a rapidity divide $\eta_{2}$ which separates the gluon field in quantum and classical. Fields having rapidity up to $\eta_{2}$ are considered classical fields while those having rapidity $\eta_{1}>\eta>\eta_{2}$ are the quantum fields over which we will functionally integrate obtaining Feynman diagrams.

The separation in quantum and classical field may be formally written as

$$
\left\langle\mathscr{O}^{\eta_{1}}\right\rangle_{A} \rightarrow\left\langle\mathscr{O}^{\prime \eta_{2}} \otimes \mathscr{O}^{\prime \eta_{1}}\right\rangle_{A}
$$

Here, the subscript $A$ indicates that the matrix elements are evaluated in the background of the gluonic external field. In principle, after the separation of the fields in classical and quantum components, the operator may be different from the one we started with. We have indicated this with a prime on the operator $\mathscr{O}$ in Eq. (4.6). Since particles with different rapidities perceive each other as Wilson lines, the operators obtained after splitting the fields in classical and quantum are still Wilson lines.

The result of the integration of the matrix element on the right-hand-side (RHS) of Eq. (4.6) over the quantum fields is the kernel of the one loop evolution equation times the matrix element of the operator made of the classical fields i.e. with rapidity parallel to $\eta_{2}$, and times the infinitesimal step in rapidity $\Delta \eta=\eta_{2}-\eta_{1}$. The one-loop evolution equation of the $\mathscr{O}$ operator with respect to rapidity is

$$
\left\langle\mathscr{O}^{\eta_{1}}\right\rangle_{A}=\alpha_{S}\left(\eta_{1}-\eta_{2}\right) K_{\mathrm{evol}} \otimes\left\langle\mathscr{O}^{\prime \eta_{2}}\right\rangle_{A}
$$

The resulting evolution equation, obtained following the semi-classical approach just described, can be linear or non-linear:

- Linear evolution equation: $\mathscr{O}^{\eta_{1}}=\alpha_{s} \Delta \eta K_{\text {evol }} \otimes \mathscr{O}^{\eta_{2}}$

- Non-linear evolution equation: $\mathscr{O}^{\eta_{1}}=\alpha_{s} \Delta \eta K_{\mathrm{evol}} \otimes\left\{\mathscr{O}^{\eta_{2}} \mathscr{O}^{\eta_{2}}\right\}$

The rapidity evolution of one Wilson line is given by the Feynman diagrams in Fig. (4). The red strip in the figure represents the background field in the spectator frame: the external field is highly boosted, and it gets contracted in the direction of the boost and time-dilated. Thus, the propagation of the particle is in the background of a shock-wave external field. The evolution 


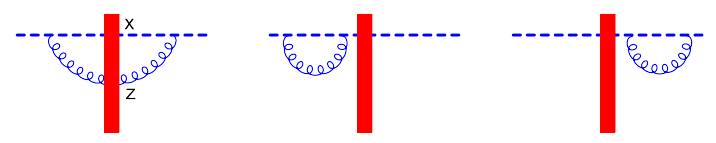

Figure 6: Feynman diagrams for one loop evolution of one Wilson lines.

equation for one Wilson line is

$$
\left\langle\left\{U_{x}^{\eta_{1}}\right\}_{i j}\right\rangle_{A}=\frac{\alpha_{s}}{2 \pi^{2}} \Delta \eta \int \frac{d^{2} z}{(x-z)_{\perp}^{2}}\left[\left\langle\operatorname{tr}\left\{U_{x}^{\eta_{2}} U_{z}^{\eta_{2} \dagger}\right\}\left\{U_{z}^{\eta_{2}}\right\}_{i j}\right\rangle_{A}-\left\langle\frac{1}{N_{c}}\left\{U_{x}^{\eta_{2}}\right\}_{i j}\right\rangle_{A}\right]
$$

where we have used the short-hand notation $U_{x} \equiv U\left(x_{\perp}\right)$, and the color index $i, j=1,2,3$. The kernel of the evolution in this case is $K(x, z)=\frac{1}{(x-z)^{2}}$, and the equation is clearly non-linear. Before the one-loop evolution, we have one Wilson line with rapidity $\eta_{1}$ corresponding to the propagation of one quark in the background of a shock-wave. At one loop order, instead, we have a quark and a gluon propagating in the shock-wave.

\section{Leading order evolution equation: the BK equation}

The scattering amplitude of DIS is proportional to

$$
\mathscr{U}\left(x_{\perp}, y_{\perp}\right)=1-\frac{1}{N_{c}} \operatorname{tr}\left\{U\left(x_{\perp}\right) U^{\dagger}\left(y_{\perp}\right)\right\}
$$

To get the evolution equation of the color-dipole we need the evolution equation of the operator $U_{x}$ and $U_{y}^{\dagger}$, and also the evolution equation of pairwise interactions. Thus, the operator $\frac{d}{d \eta}$ of the evolution equation does not follow the Leibniz rule for derivative of product of function. Indeed, this is represented by the Feynman diagrams in Fig. (5),

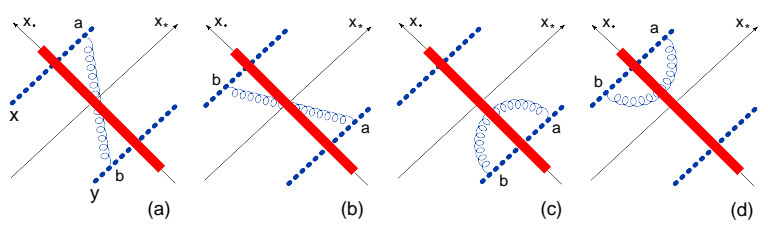

Figure 7: Feynamn diagram for the LO evolution equation of a color-dipole. We omitted the virtual correction diagrams.

and the corresponding evolution equation is

$$
\frac{d}{d \eta} \hat{\mathscr{U}}(x, y)=\frac{\alpha_{s} N_{c}}{2 \pi^{2}} \int \frac{d^{2} z(x-y)^{2}}{(x-z)^{2}(y-z)^{2}}\{\hat{\mathscr{U}}(x, z)+\hat{\mathscr{U}}(z, y)-\hat{\mathscr{U}}(x, y)-\hat{\mathscr{U}}(x, z) \hat{\mathscr{U}}(z, y)\}
$$

Equation (5.2) is the LO Balitsky evolution equation [4] for color dipole. When the non-linear term operator $\langle\hat{\mathscr{U}}(x, z) \hat{\mathscr{U}}(z, y)\rangle$ factorizes at large $N_{c}$ as $\langle\hat{\mathscr{U}}(x, z)\rangle\langle\hat{\mathscr{U}}(z, y)\rangle$, Eq. (5.2) becomes the Balitsky-Kovchegov equation [4,5]. The linear terms in Eq. (5.2) correspond to the BFKL evolution equation obtained in perturbative QCD in the leading-log resummation $\alpha \ll 1$ and $\alpha_{s} \eta \sim 1$; 
while the non-linear term appears because of the semi-classical approach where the new resummation parameter is $\alpha_{s}^{2} A^{1 / 3}$ with $A$ being the atomic number in the case of DIS off a nuclear target. The BFKL equation is known to violate unitarity, but the non linear term in Eq. (5.2) preserves unitarity.

In order to get the LO evolution equation for trace of any number of Wilson lines or product of any number of Wilson lines, which would correspond to the Balitsky-JIMWLK evolution equation $[4,6]$, one needs to obtain the evolution equation for the following operators as well: $\left\{U_{x}^{\dagger \eta_{1}}\right\}_{i j},\left\{U_{x}^{\eta_{1}} U_{y}^{\eta_{1}}\right\}_{i j},\left\{U_{x}^{\eta_{1}} U_{y}^{\dagger \eta_{1}}\right\}_{i j},\left\{U_{x}^{\dagger \eta_{1}} U_{y}^{\dagger \eta_{1}}\right\}_{i j}$. Thus, one obtains a set of five evolution equations that can be used to calculate the evolution equation of operators with any trace of Wilson lines. As an example, let us consider the evolution equation of a four Wilson lines operator $\operatorname{tr}\left\{U_{x} U_{y}^{\dagger} U_{w} U_{z}^{\dagger}\right\}$. To this end, one has to sum the evolution of each single Wilson line using the evolution equation like Eq. (4), and similar evolution equations for each paring.

Our next task is to obtain the evolution equations of matrix elements of operators with any trace of Wilson lines at NLO. We will obtain evolution equations similar to the one given in (4.8) but at the next-to-leading order (NLO).

\section{Next-to-leading order evolution equation}

In order to obtain the evolution equation of operators with any trace of Wilson lines (or also product of Wilson lines) at the next to leading order, one has to calculate a similar set of evolution equations at NLO. At NLO, however, we may have not only the evolution of single Wilson lines and of two connected Wilson lines but also evolution of triple Wilson lines interaction (see Fig. 6 e) and f)). The diagrams contributing to the NLO B-JIMWLK with three Wilson lines were calculated
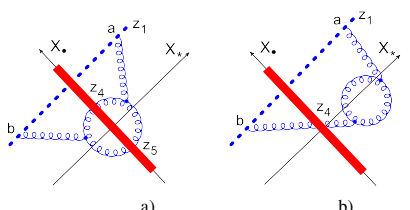

b)

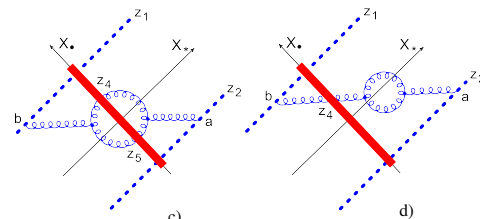

c)

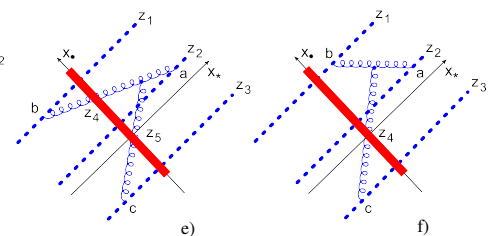

Figure 8: Sample of Feynman diagrams contributing to the NLO B-JIMWLK evolution equation.

in Ref. [10]. In Ref. [9] we have calculated the full Balitsky hierarchy at NLO, confirming also the result obtained in Ref. [10]. The NLO JIMWLK Hamiltonian [11], on the other hand, was obtained using the NLO BK equation calculated in Ref. [12,13] and the evolution with three connected Wilson lines of Ref. [10].

For simplicity, we present here only the one-particle interaction ("gluon reggeization" term) at NLO

$$
\begin{aligned}
& \frac{d}{d \eta}\left(U_{1}\right)_{i j}=\frac{\alpha_{s}^{2}}{8 \pi^{4}} \int \frac{d^{2} z_{4} d^{2} z_{5}}{z_{45}^{2}}\left\{U _ { 4 } ^ { d d ^ { \prime } } ( U _ { 5 } ^ { e e ^ { \prime } } - U _ { 4 } ^ { e e ^ { \prime } } ) \left(\left[2 I_{1}-\frac{4}{z_{45}^{2}}\right] f^{a d e} f^{b d^{\prime} e^{\prime}}\left(t^{a} U_{1} t^{b}\right)_{i j}+\frac{\left(z_{14}, z_{15}\right)}{z_{14}^{2} z_{15}^{2}} \ln \frac{z_{14}^{2}}{z_{15}^{2}}\right.\right. \\
& \left.\left.\times\left[i f^{a d^{\prime} e^{\prime}}\left(\left\{t^{d}, t^{e}\right\} U_{1} t^{a}\right)_{i j}-i f^{a d e}\left(t^{a} U_{1}\left\{t^{d^{\prime}}, t^{e^{\prime}}\right\}\right)_{i j}\right]\right)\right\} \\
& +\frac{\alpha_{s}^{2} N_{c}}{4 \pi^{3}} \int d^{2} z_{4} z_{14}^{2}\left(U_{4}^{a b}-U_{1}^{a b}\right)\left(t^{a} U_{1} t^{b}\right)_{i j}\left\{\left[\frac{11}{3} \ln z_{14}^{2} \mu^{2}+\frac{67}{9}-\frac{\pi^{2}}{3}\right]\right\}
\end{aligned}
$$


where

$$
I_{1} \equiv I\left(z_{1}, z_{4}, z_{5}\right)=\frac{\ln z_{14}^{2} / z_{15}^{2}}{z_{14}^{2}-z_{15}^{2}}\left[\frac{z_{14}^{2}+z_{15}^{2}}{z_{45}^{2}}-\frac{\left(z_{14}, z_{15}\right)}{z_{14}^{2}}-\frac{\left(z_{14}, z_{15}\right)}{z_{15}^{2}}-2\right]
$$

and the evolution for triple interactions (a sample of diagrams is given in Fig. 3 e and f).

$$
\begin{aligned}
& \frac{d}{d \eta}\left(U_{1}\right)_{i j}\left(U_{2}\right)_{k l}\left(U_{3}\right)_{m n}=i \frac{\alpha_{s}^{2}}{2 \pi^{4}} \int d^{2} z_{4} d^{2} z_{5}\left\{\mathscr{J}_{12345} \ln \frac{z_{34}^{2}}{z_{35}^{2}}\right. \\
& \times f^{c d e}\left[\left(t^{a} U_{1}\right)_{i j}\left(t^{b} U_{2}\right)_{k l}\left(U_{3} t^{c}\right)_{m n}\left(U_{4}-U_{1}\right)^{a d}\left(U_{5}-U_{2}\right)^{b e}\right. \\
& \left.-\left(U_{1} t^{a}\right)_{i j}\left(U_{2} t^{b}\right)_{k l}\left(t^{c} U_{3}\right)_{m n}\left(U_{4}-U_{1}\right)^{d a}\left(U_{5}-U_{2}\right)^{e b}\right] \\
& +\mathscr{J}_{32145} \ln \frac{z_{14}^{2}}{z_{15}^{2}} f^{a d e}\left[\left(U_{1}^{\dagger} t^{a}\right)_{i j}\left(t^{b} U_{2}\right)_{k l}\left(t^{c} U_{3}\right)_{m n}\left(U_{4}-U_{3}\right)^{c d}\left(U_{5}-U_{2}\right)^{b e}\right. \\
& \left.-\left(t^{a} U_{1}\right)_{i j} \otimes\left(U_{2} t^{b}\right)_{k l}\left(U_{3} t^{c}\right)_{m n}\left(U_{4}^{d c}-U_{3}^{d c}\right)\left(U_{5}^{e b}-U_{2}^{e b}\right)\right] \\
& +\mathscr{J}_{13245} \ln \frac{z_{24}^{2}}{z_{25}^{2}} f^{b d e}\left[\left(t^{a} U_{1}\right)_{i j}\left(U_{2}^{\dagger} t^{b}\right)_{k l}\left(t^{c} U_{3}\right)_{m n}\left(U_{4}-U_{1}\right)^{a d}\left(U_{5}-U_{2}\right)^{c e}\right. \\
& \left.-\left(U_{1} t^{a}\right)_{i j}\left(t^{b} U_{2}\right)_{k l}\left(U_{3} t^{c}\right)_{m n}\left(U_{4}-U_{1}\right)^{d a}\left(U_{5}-U_{3}\right)^{e c}\right]
\end{aligned}
$$

where

$$
\begin{aligned}
& \mathscr{J}_{12345} \equiv \mathscr{J}\left(z_{1}, z_{2}, z_{3}, z_{4}, z_{5}\right)=-\frac{2\left(z_{14}, z_{34}\right)\left(z_{25}, z_{35}\right)}{z_{14}^{2} z_{25}^{2} z_{34}^{2} z_{35}^{2}} \\
& -\frac{2\left(z_{14}, z_{45}\right)\left(z_{25}, z_{35}\right)}{z_{14}^{2} z_{25}^{2} z_{35}^{2} z_{45}^{2}}+\frac{2\left(z_{25}, z_{45}\right)\left(z_{14}, z_{34}\right)}{z_{14}^{2} z_{25}^{2} z_{34}^{2} z_{45}^{2}}+\frac{\left(z_{14}, z_{25}\right)}{z_{14}^{2} z_{25}^{2} z_{45}^{2}}
\end{aligned}
$$

Here we have used the short-hand notation $U_{1} \equiv U\left(z_{1}\right)$ etc. In Eq. (6.1) we have omitted the quark-loop contributions. The evolution for pairwise interactions at NLO can be found in Ref. [9].

\section{Conclusions}

Non-linear dynamics in QCD scattering processes is nowadays studied in several experiments around the world and it is subject for future experiments like the Electron-Ion-Collider.

Suitable operators for the description of QCD non-linear dynamics are Wilson lines operators. The OPE at high-energy in terms of Wilson lines allows one to factorize the amplitude in rapidity space. The validity of this factorization has been proven at NLO accuracy [1,2]. We have seen that the first of the Balitsky's hierarchy equations reduces to the BK equation in the large $N_{c}$ limit. To obtain the extension of the NLO BK equation to the NLO evolution of any number of Wilson lines with open color indexes, we had to consider the triple interaction type of diagrams as well as the self-energy and pairwise interaction. We have presented here only the NLO evolution equation for self-energy interactions given in Eq. (6.1) and the evolution equation of Wilson lines with triple interactions given in Eq. (6.2). The evolution equation for pairwise interactions at NLO can be found in Ref. [9].

This material is based upon work supported by the U.S. Department of Energy, Office of Science, Office of Nuclear Physics under Award Number DE-SC0004286. 


\section{References}

[1] I. Balitsky and G. A. Chirilli, Phys. Rev. D 87, 014013 (2013) [arXiv:1207.3844 [hep-ph]].

[2] I. Balitsky and G. A. Chirilli, Phys. Rev. D 83, 031502 (2011) [arXiv:1009.4729 [hep-ph]].

[3] G. A. Chirilli and Y. V. Kovchegov, JHEP 1405, 099 (2014) [JHEP 1508, 075 (2015)]

[arXiv:1403.3384 [hep-ph]].

[4] I. Balitsky, Nucl. Phys. B 463, 99 (1996) [hep-ph/9509348].

[5] Y. V. Kovchegov, Phys. Rev. D 60, 034008 (1999) [hep-ph/9901281].

[6] J. Jalilian Marian, A. Kovner, A.Leonidov and H. Weigert, Nucl. Phys. B504, 415 (1997), Phys. Rev. D59, 014014 (1999); J. Jalilian Marian, A. Kovner and H. Weigert, Phys. Rev. D59, 014015 (1999); H. Weigert, Nucl. Phys. A703, 823 (2002); E.Iancu, A. Leonidov and L. McLerran, Nucl. Phys. A692, 583 (2001), Phys. Lett. B510, 133 (2001).

[7] I. Balitsky and G. A. Chirilli, Phys. Lett. B 687, 204 (2010) [arXiv:0911.5192 [hep-ph]].

[8] I. Balitsky, In *Shifman, M. (ed.): At the frontier of particle physics, vol. 2* 1237-1342 [hep-ph/0101042].

[9] I. Balitsky and G. A. Chirilli, Phys. Rev. D 88, 111501 (2013) [arXiv:1309.7644 [hep-ph]].

[10] A. V. Grabovsky, JHEP 1309, 141 (2013) [arXiv:1307.5414 [hep-ph]].

[11] A. Kovner, M. Lublinsky and Y. Mulian, Phys. Rev. D 89, 061704 (2014) [arXiv:1310.0378 [hep-ph]].

[12] I. Balitsky and G. A. Chirilli, Phys. Rev. D 77, 014019 (2008) [arXiv:0710.4330 [hep-ph]].

[13] I. Balitsky and G. A. Chirilli, Nucl. Phys. B 822, 45 (2009) [arXiv:0903.5326 [hep-ph]]. 